\title{
Is obstructive sleep apnoea an innocent bystander in the pathophysiology of arterial stiffening?
}

\author{
Craig L Phillips, ${ }^{1,2,3}$ Luciano F Drager $^{4,5}$
}

Arterial compliance is an important mechanical property of the arterial tree that is crucial for the maintenance of vascular homeostasis and cardiovascular health. There are a number of factors that variably decrease arterial compliance and therefore increase arterial stiffness in different arterial segments. For example, arteries become less distensible and therefore stiffer as blood pressure (BP) increases, and this effect occurs throughout the arterial tree. On the other hand, an increase in the collagen-elastin ratio in the arterial wall, such as occurs with ageing, increases arterial stiffness predominantly in large elastic arteries like the aorta. In contrast, an increase in the quantity and tone of arterial smooth muscle mainly increases arterial stiffness in the smaller conduit arteries such as the femoral arteries. $^{12}$

Overall, a loss of arterial compliance gives rise to increased arterial stiffness and poorer maintenance of vascular homeostasis, and this is likely to be a key driver of cardiovascular disease (CVD). The process of arterial stiffening occurs in ageing populations and in conditions that are strongly associated with CVD including chronic kidney disease, ${ }^{3}$ type II diabetes and various components of the metabolic syndrome. ${ }^{4}$ This has led to the development of many methods for quantifying arterial stiffness, ${ }^{5}$ as a means of improving CVD risk stratification in populations with comorbid disease. However, in order to standardise the assessment of risk, the American Heart Association Council for high blood pressure research recommends the non-invasive

${ }^{1}$ CIRUS, Centre for Sleep and Chronobiology, Woolcock Institute of Medical Research, University of Sydney, Sydney, New South Wales, Australia

${ }^{2}$ Department of Respiratory and Sleep Medicine, Royal North Shore Hospital, St Leonards, New South Wales, Australia

${ }^{3}$ Sydney Medical School, University of Sydney, Sydney, New South Wales, Australia

${ }^{4}$ Hypertension Unit, Heart Institute (InCor), University of Sao Paulo Medical School, Sao Paulo, Brazil

${ }^{5}$ Hypertension Unit, Renal Division, University of Sao Paulo Medical School, Sao Paulo, Brazil

Correspondence to Associate Professor Craig L Phillips, Woolcock Institute of Medical Research, PO Box M77, Missenden Road, NSW 2050, Australia; craig.phillips@sydney.edu.au measurement of the carotid-femoral pulse wave velocity as the preferred method. ${ }^{6}$ Studies have established that an increase in pulse wave velocity (PWV) of $1 \mathrm{~m} / \mathrm{s}$ increases CVD events by $14 \%$ and CVD mortality by $15 \%$.

Obstructive sleep apnoea (OSA) has in recent years been increasingly linked with an increased prevalence and incidence of CVD independent of traditional risk factors including age and obesity. ${ }^{8}$ In this context, numerous studies have demonstrated an association between OSA and increased arterial stiffness ${ }^{5}$ with some studies explicitly demonstrating additional (independent) effects beyond classical risk factors that promote arterial stiffening, such as the metabolic syndrome. ${ }^{9}$ Furthermore, several observational studies have shown a reduction in a range of arterial stiffness measurements following treatment of OSA with nasal CPAP. ${ }^{5}$ However, the majority of studies have only included small numbers, and most were of short duration. In addition, most were uncontrolled observational studies. Among the few randomised controlled trials (RCT), some $\mathrm{e}^{10-12}$ but not all, ${ }^{13} 14$ demonstrated reductions in measures of arterial stiffness with CPAP. This lack of a consistent effect with CPAP may be due to a number of factors including small sample sizes, variability in patient characteristics including presence versus absence of comorbid risk factors, elevated versus normal baseline BP and arterial stiffness, variability in CPAP compliance and length of CPAP treatment and variability in the methods of arterial stiffness assessment. Nevertheless, the lack of OSA treatment does not appear to reduce the effectiveness of antihypertensive treatment in reducing 24 hour BP and PWV in hypertensive patients, suggesting that it is not a major driver of arterial stiffness. ${ }^{15}$ Therefore, it still remains unclear whether OSA can be truly implicated as a causal factor for increased arterial stiffness or whether it is in fact an innocent bystander. Given the high prevalence in OSA of comorbid conditions that are well proven to promote arterial stiffening including obesity, hypertension, dyslipidaemia, glucose intolerance and type II diabetes, it is important that further studies involving larger samples of patients are conducted to address the 'uncertainty of causality'.

In an effort to address this, Joyeux-Faure et $a l^{16}$ report on the results of a meta-analysis to explore whether and to what extent OSA 'drives' arterial stiffness. They used individual data from 893 patients (72\% men, mean age $56 \pm 11$ years) who participated in nine statistically homogeneous studies conducted between 2006 and 2015 at their institution. Six of the nine studies included analysis of baseline data from randomised trials. All subjects were well characterised in terms of anthropometric variables (age, body mass index, gender) and other comorbidities (BP, antihypertensive use, diabetic and lipid lowering medications). OSA was assessed either by full polysomnography or respiratory polygraphy and confirmed in $84 \%$ of patients. All patients underwent carotid-femoral PWV assessment using a standardised protocol with the same Complior (Alam Medical, France) device. The sample included a wide spectrum of OSA and obesity severities as well as a moderate to high prevalence of hypertension (78\%), type 2 diabetes $(45 \%)$ and dyslipidaemia (62\%) which are known drivers for increased arterial stiffness. PWV varied widely among patients $(5.3 \mathrm{~m} / \mathrm{s}$ to $20.5 \mathrm{~m} / \mathrm{s})$ with a mean $( \pm S D)$ value of $10.4 \pm 2.3 \mathrm{~m} / \mathrm{s}$. This contrasts with an expected mean PWV ( $\pm 2 \mathrm{SD})$ of $8.3 \mathrm{~m} / \mathrm{s}$ (4.5-12.1) in similarly aged people who have normal BP and no additional cardiovascular risk factors. ${ }^{17}$

As expected, the initial univariate analysis found that increased PWV was strongly associated with the traditional risk factors of age, BP, diabetes and dyslipidaemia. However, neither OSA-related hypoxia nor severe OSA (defined by an Apnoea Hypopnea Index (AHI) $\geq 30$ events/hour) was associated with increased PWV. Interestingly, subjective sleepiness defined by an Epworth Sleepiness Scale (ESS) score $\geq 9$ (which was highest in patients with severe OSA) was associated with a reduced PWV, suggesting that sleepiness may have confounded the effects in patients with severe OSA. However, ultimately in the final multivariable analysis, only age, systolic BP and type 2 diabetes remained significantly associated with increased PWV.

The authors acknowledged several limitations of their analysis. Heterogeneity might have been introduced with the mixed use of polygraphy and polysomnography across studies and by including studies with different eligibility criteria. Use of polygraphy could dilute the difference in PWV attributable to severe OSA due to the increased probability of diluting OSA severity in 
many patients secondary to poor sleep on the night. There was also significant hypoxia in the control (non-OSA) group due to COPD or morbid obesity which may have also diluted the OSA effect. In addition to these acknowledged limitations, it is important to recognise that all patients were recruited on the basis of suspected OSA, most (72\%) were men, all were from a single centre, and it was unclear whether the population was predominantly of the same ethnicity. These factors could all make the findings non-generalisable. In this sense, collaborative databank sharing comprising individual data from international studies that used similar PWV techniques would be more appropriate to prevent this limitation. Furthermore, the study contained only 58 patients $(\sim 6 \%)$ without OSA (AHI <5) which effectively limits the analysis almost entirely to an OSA population in which CVD risk factors are notoriously common, and this clinical referral bias could increase the likelihood of a null finding. In this context, all OSA-related variables were analysed as dichotomous variables using cut points instead of continuous variables, and this approach may have reduced the power to detect true associations.

Regardless of the aforementioned potential limitations, the analysis should be recognised for its valuable contribution to the evidence base which to date has been limited for the reasons previously stated. There was adequate power to rigorously explore the independent association between OSA and increased arterial stiffness measured in a standardised way using the gold standard carotid-femoral PWV. Whether OSA contributes to arterial stiffness in the more muscular conduit arteries was not able to be determined, and hopefully future adequately powered analyses will be able to explore this. Finally, it is important to highlight that while the analysis did aim to explore the extent to which OSA 'drives' arterial stiffness, causality cannot be inferred from this cross-sectional data. Further analyses of data from randomised trials are needed. In this context, it would be interesting to see whether individual patient data from the RCT studies included in this analysis would shed light on a causal association.

Contributors Both authors contributed equally to the work.

Funding The authors have not declared a specific grant for this research from any funding agency in the public, commercial or not-for-profit sectors.

Competing interests None declared.

Patient consent Not required.

Provenance and peer review Commissioned; internally peer reviewed.

(c) Author(s) (or their employer(s)) 2018. No commercial re-use. See rights and permissions. Published by BMJ.

\section{Check for updates}

To cite Phillips CL, Drager LF. Thorax 2018;73:1099-1100.

Accepted 20 August 2018

Published Online First 10 August 2018

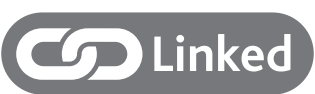

- http://dx.doi.org/10.1136/thoraxjnl-2018-211513

Thorax 2018;73:1099-1100.

doi:10.1136/thoraxjnl-2018-212332

\section{REFERENCES}

1 Safar ME, Blacher J, Jankowski P, et al. Arterial stiffness, pulse pressure, and cardiovascular disease-is it possible to break the vicious circle? Atherosclerosis 2011;218:263-71

2 Lacolley P, Regnault V, Segers P, et al. Vascular smooth muscle cells and arterial stiffening: relevance in development, aging, and disease. Physiol Rev 2017:97:1555-617.

3 Townsend RR. Arterial stiffness in CKD: a review. Am J Kidney Dis 2018.

4 Smulyan H, Lieber A, Safar ME, et al. Hypertension, diabetes type II, and their association: role of arterial stiffness. Am J Hypertens 2016;29:5-13.
5 Phillips CL, Butlin M, Wong KK, et al. Is obstructive sleep apnoea causally related to arterial stiffness? A critical review of the experimental evidence. Sleep Med Rev 2013:17:7-18.

6 Townsend RR, Wilkinson IB, Schiffrin EL, et al. Recommendations for Improving and Standardizing Vascular Research on Arterial Stiffness: A Scientific Statement From the American Heart Association. Hypertension 2015;66:698-722.

7 Vlachopoulos C, Aznaouridis K, Stefanadis C, et al. Prediction of cardiovascular events and all-cause mortality with arterial stiffness: a systematic review and meta-analysis. J Am Coll Cardiol 2010:55:1318-27.

8 Drager LF, McEvoy RD, Barbe F, et al. Sleep apnea and cardiovascular disease: lessons from recent trials and need for team science. Circulation 2017; 136:1840-50.

9 Drager LF, Bortolotto LA, Maki-Nunes C, et al. The incremental role of obstructive sleep apnoea on markers of atherosclerosis in patients with metabolic syndrome. Atherosclerosis 2010;208:490-5.

10 Drager LF, Bortolotto LA, Figueiredo AC, et al. Effects of continuous positive airway pressure on early signs of atherosclerosis in obstructive sleep apnea. Am J Respir Crit Care Med 2007;176:706-12.

11 Kohler M, Pepperell JC, Casadei B, et al. CPAP and measures of cardiovascular risk in males with OSAS. Eur Respir J 2008:32:1488-96.

12 Litvin AY, Sukmarova ZN, Elfimova EM, et al. Effects of CPAP on "vascular" risk factors in patients with obstructive sleep apnea and arterial hypertension. Vasc Health Risk Manag 2013;9:229-35.

13 Hoyos CM, Yee BJ, Wong KK, et al. Treatment of sleep apnea with CPAP lowers central and peripheral blood pressure independent of the time-of-day: a randomized controlled study. Am J Hypertens 2015;28:1222-8.

14 Jones $\mathrm{A}$, Vennelle M, Connell M, et al. The effect of continuous positive airway pressure therapy on arterial stiffness and endothelial function in obstructive sleep apnea: a randomized controlled trial in patients without cardiovascular disease. Sleep Med 2013:14:1260-5.

15 Fatureto-Borges F, Jenner R, Costa-Hong V, et al. Does obstructive sleep apnea influence blood pressure and arterial stiffness in response to antihypertensive treatment? Hypertension 2018:72:399-407.

16 Joyeux-Faure M, Tamisier R, Borel JC, et al. Contribution of obstructive sleep apnoea to arterial stiffness: a meta-analysis using individual patient data. Thorax 2018:thoraxinl-2018-211513.

17 The Reference Values for Arterial Stiffness' Collaboration. Determinants of pulse wave velocity in healthy people and in the presence of cardiovascular risk factors: 'establishing normal and reference values'. Eur Heart J 2010;31:2338-50. 